\title{
An Exact Algorithm for Minimum Distortion Embedding
}

\author{
Fedor V. Fomin* Daniel Lokshtanov* Saket Saurabh*
}

\begin{abstract}
Let $G$ be an unweighted graph on $n$ vertices. We show that an embedding of the shortest path metric of $G$ into the line with minimum distortion can be found in time $5^{n+o(n)}$. This is the first algorithm breaking the trivial $n$ !-barrier.
\end{abstract}

\section{Introduction}

Given an undirected graph $G$ with the vertex set $V(G)$ and the edge set $E(G)$, the graph metric of $G$ is $M(G)=\left(V(G), D_{G}\right)$, where the distance function $D_{G}$ is the shortest path distance between $u$ and $v$ for every pair of vertices $u, v \in V(G)$. Given a graph metric $M$ and another metric space $M^{\prime}$ with distance functions $D$ and $D^{\prime}$, a mapping $f: M \rightarrow M^{\prime}$ is called an embedding of $M$ into $M^{\prime}$. The mapping $f$ has contraction $c_{f}$ and expansion $e_{f}$ if for every pair of points $p, q$ in $M$,

$$
D(p, q) \leq D^{\prime}(f(p), f(q)) \cdot c_{f},
$$

and

$$
D(p, q) \cdot e_{f} \geq D^{\prime}(f(p), f(q))
$$

respectively. We say that $f$ is non-contracting if $c_{f}$ is at most 1. A non-contracting mapping $f$ has distortion $d$ if $e_{f}$ is at most $d$.

In this paper we provide an exact algorithm for the following fundamental problem: For a given graph $G$, find a minimum distortion embedding of the graph metric of $G$ into the line. In this case the metric space $M^{\prime}$ is $\mathbb{R}^{1}$ and $D^{\prime}$ is the Euclidean distance. A simple algorithm is to try all possible permutations of the vertex set. Each permutation corresponds to an embedding where the distance between two consecutive vertices on the line is equal to the shortest path distance between them. The running time this algorithm is $O(n ! n)$ and to the best of our knowledge, no faster exact algorithm for any kind of embedding problem was known prior to our work.

The problem of finding an embedding with low distortion between metric spaces is a fundamental mathematical problem $[8,10]$ that has been studied intensively. Embedding a graph metric into a simple low-dimensional metric space like the real line has proved to be a useful algorithmic tool in various fields. A long list of applications given in [7] includes approximation algorithms for graph and network problems, such as sparsest cut, minimum bandwidth, low-diameter decomposition and optimal group Steiner trees, and online algorithms for metrical task systems and file migration problems. The algorithmic issues of metric embeddings has recently begun to develop [1, 2, 3, 9]. For example, Bădoiu et al. [1] describe approximation algorithms and hardness results for embedding general metrics

\footnotetext{
*Department of Infomatics, University of Bergen, Norway. \{fedor.fomin|daniellolsaket\}@ii.uib.no.
} 
into the line. In particular they show that the minimum distortion for a line embedding is hard to approximate up to a factor polynomial in $n$, even for weighted trees where the ratio of maximum/minimum weights is bounded by a polynomial in $n$. For the case of unweighted graphs, it was shown by Bădoiu et al. [2] that there is a constant $a>1$, such that $a$ approximation of the minimum distortion of an embedding into the line is $N P$-hard. Bădoiu et al. also provided an exact algorithm for computing an embedding with distortion at most $d$ in time $n^{O(d)}$. For $d=\Omega(n)$ the running time of such an algorithm is $n^{O(n)}$. Fellows et al. [6] studied the parameterized complexity of metric embeddings and proved that embedding into the line and more generally, into trees with bounded vertex degrees, is fixed parameter tractable when parameterized by the distortion. For embedding a graph metric into the line the running time of the algorithm described in [6] is $O\left(n d^{4}(2 d+1)^{2 d}\right)$, which also does not break the barrier of $n$ ! when $d=\Omega(n)$.

It is worth to mention the resemblance between the problem of embedding into the line and the Bandwidth Minimization problem. In the Bandwidth Minimization problem the objective is for a given graph $G$ to find a bijective mapping $f: V(G) \rightarrow\{1, \ldots, n\}$, for which the bandwidth, that is $b=\max _{(u, v) \in E(G)}|f(u)-f(v)|$, is minimized. Observe that the only difference between the two problems is that in the BANDWIDTH Minimization problem we demand $1 \leq|f(p)-f(q)|$ for every pair of vertices while the non-contraction constraint in our embedding problem is $D(p, q) \leq|f(p)-f(q)|$.

The Bandwidth Minimization problem is one of the test-bed problems in the area of moderately exponential time algorithms and has been studied intensively. Trying all possible permutations of the vertex set yields a simple $O(n ! n)$ time algorithm while the known algorithms for the problem with running time $O\left(c^{n}\right)$ are far from straightforward. The $O(n$ !)-barrier was broken by Feige and Kilian [5] who gave an algorithm with running time $10^{n} n^{O(1)}$. This result was subsequently improved by Cygan and Pilipczuk [4] down to $5^{n} n^{O(1)}$.

Despite the similarities between low distortion embedding into the line and bandwidth, the non-contraction constraint makes the algorithmic complexity of the two problems significantly different. A striking example is that the parameterized version of the BANDWIDTH Minimization problem is one of the hardest problems in Parameterized Complexity, while low distortion embedding into the line is fixed parameter tractable [6]. Thus, it is not surprising that a direct transmission of the ideas for the Bandwidth Minimization problem to low distortion embeddings does not work. Nevertheless, our approach is still based on the approaches from $[4,5]$, especially the initial and final parts of our algorithm. However, to handle non-contraction need a non-trivial additional link connecting these parts.

\section{Preliminaries}

Let $G$ be an undirected graph with vertex set $V(G)$ and edge set $E(G)$. We denote the number of vertices by $n$. For $u$ and $v \in V(G), D_{G}(u, v)$ is the shortest path distance between $u$ and $v$ in $G$. For a subset $V^{\prime} \subseteq V(G)$, by $G\left[V^{\prime}\right]$ we mean the subgraph of $G$ induced by $V^{\prime}$. An integer interval is a set $\{x, x+1, \ldots, y-1, y\}$ of integers appearing consecutively. An embedding of a graph $G$ into the line is a function $f: V(G) \rightarrow \mathbb{R}$. The distortion of an embedding $f$ is $\max _{u, v \in V(G)} \frac{|f(u)-f(v)|}{D_{G}(u, v)}$. An embedding is called non-contracting if $|f(u)-f(v)| \geq D_{G}(u, v)$ for every pair $u, v$ of vertices. If $f$ is non-contracting we say that a vertex $u$ pushes vertex $v$ if $D_{G}(u, v)=|f(u)-f(v)|$. For an embedding $f$, let $v_{1}, v_{2}, \ldots, v_{n}$ 
be an ordering of the vertices such that $f\left(v_{1}\right)<f\left(v_{2}\right)<\cdots<f\left(v_{n}\right)$. We say that $f$ is pushing if $v_{i}$ pushes $v_{i+1}$, for each $1 \leq i \leq n-1$.

A partial embedding of $G$ into the line is a function $f^{\prime}: V^{\prime} \rightarrow \mathbb{R}$ for some subset $V^{\prime}$ of $V$. For a partial embedding $f^{\prime}$ with domain $V^{\prime}$, let $v_{1}^{\prime}, v_{2}^{\prime}, \ldots, v_{n^{\prime}}^{\prime}$ be an ordering of $V^{\prime}$ such that $f^{\prime}\left(v_{1}^{\prime}\right)<f^{\prime}\left(v_{2}^{\prime}\right)<\cdots<f\left(v_{n^{\prime}}^{\prime}\right)$. We say that $f^{\prime}$ is pushing if $v_{i}^{\prime}$ pushes $v_{i+1}^{\prime}$, for each $1 \leq i \leq$ $n^{\prime}-1$. The distortion of a pushing partial embedding $f^{\prime}$ is $\max _{u v \in E\left(G\left[V^{\prime}\right]\right)}\left|f^{\prime}(u)-f^{\prime}(v)\right|$.

\section{Exact Algorithm for Distortion}

In this section we give an exact algorithm for the following problem.

Given an input graph $G$ with the vertex set $V(G)$ and the edge set $E(G)$, find a mapping $f$ from $V(G) \rightarrow \mathbb{R}^{+}$such that for all $u, v \in V(G),|f(u)-f(v)| \geq$ $D_{G}(u, v)$ and the function

$$
\operatorname{dist}(G)=\max _{u, v \in V(G)} \frac{|f(u)-f(v)|}{D_{G}(u, v)}
$$

is minimized.

In order to reduce the search space we apply a simple lemma proved in [6] on minimum distortion embedding of graphs into the line.

Lemma $1([6])$

- If $G$ can be embedded into the line with distortion d, then there is a pushing embedding of $G$ into the line with distortion d. Furthermore, every pushing embedding of $G$ into the line is non-contracting.

- Let $f$ be a pushing embedding of a connected graph $G$ into the line with distortion at most $d$. Then $D\left(v_{i-1}, v_{i}\right) \leq d$ for every $1 \leq i \leq n$.

Lemma 1 implies that it is sufficient to look for an optimal pushing embedding. Notice that a pushing embedding with $f\left(v_{1}\right)=0$ maps every vertex to an integer coordinate. Therefore we can without loss of generality restrict ourself to functions $f: V(G) \rightarrow\{0, \ldots, d n\}$. We also assume that our input graph $G$ is connected, because otherwise some pair of vertices have infinite distance between them and hence there is no non-contracting embedding of $G$ into the line.

We now present an algorithm that decides whether there is an embedding of distortion at most $d$ for the input graph $G$. It is well known that any graph $G$ with $n$ vertices can be embedded into the line with distortion at most $2 n-1$ [2]. Thus, if we want to find the minimum $d$ such that there is an embedding of $G$ into the line with distortion at most $d$ it is sufficient to try all values between 1 and $2 n-1$ for $d$. Next we describe the three main components of our algorithm and show how to combine them in order to obtain an algorithm running in time $5^{n+o(n)}$ and using $2^{n+o(n)}$ space. The first and third part of our algorithm go along the lines of the known algorithms for BANDwIDTH $[5,4]$. While these two parts are sufficient to compute bandwidth, in order to solve our problem we need an intermediate divide and conquer step to bridge the first and last part. 


\subsection{Fixing an assignment into buckets}

The algorithm loops over all possible distributions of the vertices into "buckets" on the integer line. The remaining two steps of the algorithm deal with finding an optimal embedding that agrees with the distribution made in the first step. Formally, we are looking for a pushing embedding $f: V(G) \rightarrow\{0, \ldots, d n\}$. A bucket assignment is a function $h: V(G) \rightarrow\{0, \ldots, n\}$ and an embedding $f: V(G) \rightarrow\{0, \ldots, d n\}$ of $G$ agrees with $h$ if for every vertex $v$ of $G$ we have $h(v)=\left\lfloor\frac{f(v)}{d+1}\right\rfloor$. For $i \geq 0$, the $i$-th bucket of $h$ (or the $i$-th bucket for short) is $\mathcal{B}_{i}=\{(d+1) i, \ldots,(d+1)(i+1)-1\}$ and the content of the $i$-th bucket is $V_{i}=\{v: h(v)=i\}$.

The outer loop of the algorithm goes over a set of bucket assignments such that if there is a pushing embedding $f: V(G) \rightarrow\{0, \ldots, d n\}$ with distortion at most $d$ then some $h$ we have looped over agrees with $f$. We guess a vertex $v$ such that $h(v)=0$ and fix a spanning tree $T$ of $G$ with $r_{T}$ as root. Once $h(p)$ has been determined for the parent $p$ of a node $u$ in $T$, we loop over all possible values of $h(u)$. If $h$ is to agree with some pushing embedding $f: V(G) \rightarrow\{0, \ldots, d n\}$ with distortion at most $d$ we have that $h(u)=h(p)-1, h(u)=h(p)$ or $h(u)=h(p)+1$ and that $h(u) \geq 0$. Since we have at most 3 possibilities for the placement of each vertex the outer loop needs only to go over at most $n \cdot 3^{n}$ different bucket assignments $h$.

\subsection{Dealing with Many Buckets}

In this section and Section 3.3, we provide an algorithm which given an initial bucket assignment $h$, decides whether there is a pushing embedding $f$ of the input graph into the line with distortion at most $d$ that agrees with $h$.

Our algorithm EXACT-DisT solves a slight modification of the problem. Input to this problem is a graph $G$, an integer $d$, a bucket assignment $h$, an interval $J=\{x, x+1, \ldots, y\}$ of integers and a function $g: V^{\prime} \rightarrow\{0, \ldots, d n\}$ for some subset $V^{\prime}$ of $V(G)$. Let $\mathcal{B}_{J}=$ $\bigcup_{j \in J} \mathcal{B}_{j}$ and $V_{J}=\bigcup_{j \in J} V_{j}$. The algorithm determines whether there is a partial pushing embedding $f: V_{J} \rightarrow \mathcal{B}_{J}$ with distortion at most $d$ such that $f$ agrees with $h$ and $f(v)=$ $g(v)$ for all vertices in $V^{\prime} \cap V_{J}$. To solve the original problem we make a call to ExACT$\operatorname{Dist}(G, d, h, J, g)$ where $J=\{0, \ldots, n\}$ and the domain $V^{\prime}$ of $g$ is empty. Before commencing with the algorithm, we perform a "sanity check". That is, given $h$ check whether it is even remotely feasible that $f$ can exist. We verify that $h$ satisfies the following properties.

- For every $i,\left|V_{i}\right| \leq d+1$.

- Similarly, for every edge $u v,|h(u)-h(v)| \leq 1$.

Indeed, if some of these cases do not hold, there is no embedding $f$ with distortion $d$ that agrees with $h$ and we can immediately answer "NO". At all later stages of the algorithm we assume that $h$ satisfies these properties. An outline of the algorithm without these preliminary steps is given in Figure 1. In Section 3.3 we will give an algorithm which implements Step 3 in time $2^{n} \cdot n^{O(b)}$ time, where $b=|J|$ is the number of buckets considered.

The idea behind the algorithm is as follows. When the number of buckets $|J|$ is large, our algorithm follows a divide-and-conquer approach and if the number of buckets is "small", that is roughly $n / \log ^{2} n$, we do dynamic programming. To deal with the large number of buckets we look for a "small balanced separator" to branch on. The first step of algorithm ExACT-Dist is based on the following lemma.

Lemma 2 Let $h$ be a bucket assignment and let $J=x, x+1, \ldots, y$ be an integer interval such that $\frac{n}{\log ^{2} n}<|J|$. Then there exists $j \in I=\left\{\frac{3 x+y}{4}+1, \ldots, \frac{x+3 y}{4}\right\}$ such that $\left|V_{j}\right| \leq 2 \log ^{2} n$. 
$\operatorname{ExACT-Dist}(G, d, h, J, g)$

(Here $d$ is the distortion, $h$ is the fixed bucket assignment, $J=\{x, \ldots, y\}$ is the set of indices of buckets and $g$ is a partial embedding of some of the vertices in the graph.)

1. If the size of $|J|>\frac{n}{\log ^{2} n}$ then find a bucket $V_{j}$ of the kind described in Lemma 2 else go to Step 3.

2. Enumerate all possible pushing partial embeddings $g_{j}: V_{j} \rightarrow \mathcal{B}_{j}$ of distortion at most $d$. For every such $g_{j}$ :

- Assign $g^{\prime}(v)=g_{j}(v)$ if $v \in V_{j}$ and $g^{\prime}(v)=g(v)$ if $v$ is in the domain of $g$. Let $J_{1}=\{x, \ldots, j-1, j\}$ and $J_{2}=\{j, j+1, \ldots y\}$. Recursively solve the subproblems Exact-Dist $\left(G, d, h, J_{1}, g^{\prime}\right)$ and $\operatorname{ExACT-Dist}\left(G, d, h, J_{2}, g^{\prime}\right)$. Return "YES" if both recursive calls return "YES".

3. In this case solve the problem using Lemma 5 of Section 3.3.

Figure 1: Description of the Algorithm

Proof: The proof follows from an averaging principle. For the sake of contradiction, let us assume that for every $j \in I,\left|V_{j}\right|>2 \log ^{2} n$. Then the total number of elements in the buckets $V_{j}$ with $j \in I$ is at least

$$
\sum_{j \in I}\left|V_{j}\right|>2 \log ^{2} n \cdot \frac{|J|}{2}>2 \log ^{2} n \cdot \frac{n}{2 \log ^{2} n}=n .
$$

But the sets $V_{j}$ are disjoint, and thus the sum does not exceed $|V(G)|=n$, which is a contradiction.

If $|J|$ is at least $n / \log ^{2} n$, the algorithm picks a bucket $\mathcal{B}_{j}$ and branches on all possible ways to lay out $V_{j}$ in $\mathcal{B}_{j}$. After this the problem breaks up into two independent subproblems $\left(G, d, h, J_{1}, g^{\prime}\right)$ and $\left(G, d, h, J_{2}, g^{\prime}\right)$, see Figure 1 . We argue that the two subproblems are indeed independent. Let $f$ be a pushing partial embedding of $V_{J}$ into $\mathcal{B}_{J}$ with distortion at most $d$ such that $f$ agrees with $h$ and coincides with $g$. This means that $f$ restricted to $V_{j}$ is a pushing partial embedding of $V_{j}$ into $\mathcal{B}_{j}$. We choose $g_{j}$ to coincide with $f$ on $V_{j}$ and define $g^{\prime}(v)=g_{j}(v)$ if $v \in V_{j}$ and $g^{\prime}(v)=g(v)$ if $v$ is in the domain of $g$, just as in step 2 of algorithm ExACT-Dist. If $J=\{x, \ldots, y\}$ then $J_{1}=\{x, \ldots, j\}$ and $J_{2}=\{j, \ldots, y\}$. Now $f$ restricted to $J_{1}$ is a pushing partial embedding from $V_{J_{1}}$ to $\mathcal{B}_{J_{1}}$ while $f$ restricted to $J_{2}$ is a pushing partial embedding from $V_{J_{2}}$ to $\mathcal{B}_{J_{2}}$.

In the other direction, let $f_{1}$ and $f_{2}$ be pushing partial embeddings from $V_{J_{1}}$ to $\mathcal{B}_{J_{1}}$ and from $V_{J_{2}}$ to $\mathcal{B}_{J_{2}}$ respectively, agreeing with $h$ and coinciding with $g^{\prime}$. Since $J=J_{1} \cup J_{2}$ and $J_{1} \cap J_{2}=\{j\}$ we can choose $f$ to be the partial embedding from $V_{J}$ to $\mathcal{B}_{J}$ that coincides with both $f_{1}$ and $f_{2}$. Since both $f_{1}$ and $f_{2}$ are pushing partial embeddings, so is $f$. Since every edge with both endpoints in $V_{J}$ has both endpoints either in $V_{J_{1}}$ or in $V_{J_{2}}$ and both $f_{1}$ and $f_{2}$ have distortion at most $d$, so does $f$.

Let $T(n, b)$ be the time required by algorithm ExACT-DisT on a $n$-vertex graph $G$ with $|J|=b$. Let $T^{*}(n)$ be the time required by algorithm ExACT-DisT on a $n$-vertex graph $G$ and with $|J|<n / \log ^{2} n$. An analysis of step 1 and 2 of algorithm ExACT-DisT yields the following recurrence. 


$$
T(n, b)=\left\{\begin{aligned}
\left(\begin{array}{c}
d+1 \\
2 \log ^{2} n
\end{array}\right)\left(2 \log ^{2} n\right) ! \cdot 2 T\left(n, \frac{3 b}{4}\right) & \text { if } b>\frac{n^{\prime}}{\log ^{2} n} \\
T^{*}(n) & \text { otherwise. }
\end{aligned}\right.
$$

Thus, since $b \leq n$ we have $T(n, b) \leq 2^{O\left(\log \frac{n}{n / \log ^{2} n}\right)} \cdot T^{*}(n)=2^{o(n)} \cdot T^{*}(n)$. In Section 3.3 we show how to implement the last step of algorithm ExACT-Dist to run in time $2^{n} n^{O(b)}$ which is at most $2^{n} \cdot 2^{o(n)}$ since $b \leq n / \log ^{2} n$. This yields a $2^{n+o(n)}$ runtime bound for algorithm EXACT-DisT and a $6^{n+o(n)}$ bound for deciding whether $G$ can be embedded into the line with distortion at most $d$. In Section 3.4 we will show that the running time of our algorithm in fact is bounded by $5^{n+o(n)}$.

\subsection{Dealing with Few Buckets}

In this section we give an algorithm which given an initial bucket assignment $h$, a partial assignment $g$, and an integer interval $J=\{x, \ldots, y\}$ with $|J|=b<n / \log ^{2} n$ decides whether there is a pushing partial embedding $f: V_{J} \rightarrow \mathcal{B}_{J}$ with distortion at most $d$, agreeing with $h$ and coinciding with partial assignment $g$. Our algorithm runs in time and space $2^{n} n^{O(b)}$.

The number of slots in $J$, that is positions in the line to where vertices can be mapped, is at most $b \cdot(d+1)$. Thus there could be many slots with no vertex mapped to them. We start our algorithm by guessing for every $j \in J$ the leftmost non-empty slot in each bucket $\mathcal{B}_{j}$ and a vertex from $V_{j}$ to be placed there. Naturally, if the layout of a bucket $\mathcal{B}_{j}$ with $j \in J$ has already been determined by $g$ our guesses must be consistent with this. For every $j \in J$, let $t_{j}$ denote the vertex guessed to be placed leftmost in bucket $j$. Also let $l_{j}$ denote the position guessed for $t_{j}$. After having made the guess we modify the problem at hand - we now look for a pushing partial embedding $f: V_{J} \rightarrow \mathcal{B}_{J}$ with distortion at most $d$, agreeing with $h$, coinciding with $g$ such that for every bucket $\mathcal{B}_{j}$ with $j \in J$, the leftmost vertex mapped to $\mathcal{B}_{j}$ is $t_{j}$, which is mapped to $l_{j}$. The number of possible guesses is bounded by $(d+1)^{b} n^{b}$.

We choose the ordering $\pi_{1}, \pi_{2}, \ldots, \pi_{\left|\mathcal{B}_{J}\right|}$ of the entries of $\mathcal{B}_{J}$ such that for every $i<j$ we have that $\pi_{i} \bmod (d+1) \leq \pi_{j} \bmod (d+1)$ and such that if $\pi_{i} \bmod (d+1)=\pi_{j} \bmod (d+1)$ then $\frac{\pi_{i}}{d+1} \leq \frac{\pi_{j}}{d+1}$. For example, if $J=3,4,5$ and $d=4$, then

$$
\pi_{1}, \ldots, \pi_{15}=15,20,25,16,21,26,17,22,27,18,23,28,19,24,29 .
$$

We call the ordering $\pi_{1}, \ldots, \pi_{\left|\mathcal{B}_{J}\right|}$ the bucket order of $\mathcal{B}_{j}$. Next we define the notion of a state.

Definition 1 A state $\zeta$ is a quadruple $(P, Q, R, p)$, where $P \subseteq V_{J}, Q \subseteq P$ is a set of vertices containing at most one vertex from each $V_{j}$ such that if $V_{j} \cap P \neq \emptyset$ then $V_{j} \cap Q \neq \emptyset$ and $t_{j} \in P, R \subseteq \mathcal{B}_{J}$, is a set of integers containing at most one integer from each bucket $\mathcal{B}_{j}$ and $p \leq|J|$ is a non-negative integer.

Let us observe that the number of states is at most $2^{n} \times n^{|J|} \times(d+1)^{|J|} \times\left|\mathcal{B}_{j}\right|$. If $Q \cap V_{j} \neq \emptyset$, then define $q_{j}$ to be the vertex in $Q \cap V_{j}$. If $R \cap \mathcal{B}_{j} \neq \emptyset$ let $r_{j}$ be the integer in $R \cap \mathcal{B}_{j}$. Next we define what it means for a state to be feasible:

Definition $2 A$ state is called feasible if there exists a partial embedding $f$ assigning the vertices of $P$ to the first $p$ positions in the bucket order such that the following condition hold:

1. For any edge $u v$ with $u \in P$ and $v \in P,|f(u)-f(v)| \leq d, f$ agrees with $h$ and coincides with $g$. 
2. If $V_{j} \cap P \neq \emptyset$, then $f\left(t_{j}\right)=l_{j}$ and $f\left(q_{j}\right)=r_{j}$. There is no vertex $v \in V_{j} \cap P$ such that $f(v)<l_{j}$ or $f(v)>r_{j}$.

3. For any bucket $V_{j}$ with $j \in J$, if $x, y \in V_{j}, f(x)<f(y)$ and no vertex is mapped by $f$ to the interval $\{f(x)+1, f(y)-1\}$, then $f(y)-f(x)=D_{G}(x, y)$;

4. If $j \in J$ and $j$ is not the largest element of $J, V_{j} \subseteq P$ and $V_{j+1} \cap P \neq \emptyset$, then $f\left(l_{j+1}\right)-f\left(r_{j}\right)=D_{G}\left(l_{j+1}, r_{j}\right)$.

The idea is to go through the slots in $J$ one by one in the bucket order and for each of them determine which vertex (if any) gets mapped by $f$ to this slot. The number $p$ denotes the position in the bucket order that we have reached. The set $P$ corresponds to the set of vertices that have already been placed. For every $j \in J, t_{j}$ and $q_{j}$ denote the vertices placed leftmost and rightmost in $\mathcal{B}_{j}$ respectively. Also $l_{j}$ and $r_{j}$ denotes the position of $t_{j}$ and $q_{j}$ in $\mathcal{B}_{j}$. Now we define the notion of a state succeeding another state.

Definition 3 Let $\zeta_{1}=\left(P_{1}, Q_{1}, R_{1}, p\right)$ and $\zeta_{2}=\left(P_{2}, Q_{2}, R_{2}, p+1\right)$ be two states. We say that $\zeta_{2}$ succeeds $\zeta_{1}$ if the following holds.

- Either $P_{2}=P_{1}$, or $P_{2}=P_{1} \cup\{v\}$.

- If $P_{1}=P_{2}$, then $Q_{1}=Q_{2}$ and $R_{1}=R_{2}$.

- If $P_{2}=P_{1} \cup\{v\}$ and $v \in V_{j}$, then $j=\left\lfloor\frac{\pi_{p+1}}{d+1}\right\rfloor$ and

1. If $v \in t_{j}$, then $l_{j}=\pi_{p+1}$. If $g(v)$ is defined then $g(v)=\pi_{p+1}$.

2. $Q_{2}=\left(Q_{1} \backslash\left\{q_{j}\right\}\right) \cup\{v\}$ and $R_{2}=\left(R_{1} \backslash\left\{r_{j}\right\}\right) \cup\left\{\pi_{p+1}\right\}$.

3. If $V_{j} \cap P_{1} \neq \emptyset$ then $\pi_{p+1}-r_{j}=D_{G}\left(v, q_{j}\right)$.

4. If $j$ is not the largest element of $J$ then $l_{j+1}-\pi_{p+1} \geq D\left(v, t_{j+1}\right)$.

5. If $j \in J$ and $j$ is not the largest element of $J, V_{j} \subseteq P_{2}$ and $V_{j+1} \cap P_{2} \neq \emptyset$ then $f\left(l_{j+1}\right)-f(v)=D_{G}\left(l_{j+1}, v\right)$

6. If $j$ is not the smallest element of $J$ then $N(v) \cap V_{j-1} \cap P_{2}=\emptyset$.

We now proceed to prove an observation that will be helpful for the correctness proof.

Lemma 3 Let $\zeta_{1}=\left(P_{1}, Q_{1}, R_{1}, p\right)$ be a feasible state and $\zeta_{2}=\left(P_{2}, Q_{2}, R_{2}, p+1\right)$ be a state that succeeds $\zeta_{1}$. Then $\zeta_{2}$ is feasible.

Proof: Since $\zeta_{1}=\left(P_{1}, Q_{1}, R_{1}, p\right)$ is feasible there is a partial embedding $f$ satisfying points 1-4 in Definition 2. If $P_{1}=P_{2}$ then $f$ satisfies the points $1-4$ for $\zeta_{2}$ as well. If $P_{2} \neq P_{1}$ then $P_{2} \backslash P_{1}$ contains a single vertex $v$. Let $f^{\prime}$ be a partial embedding assigning the vertices of $P_{2}$ to the first $p+1$ positions in the bucket order such that $f^{\prime}$ and $f$ coincide and $f^{\prime}(v)=\pi_{p+1}$. By point 1 of the definition of succession $f^{\prime}$ agrees with $h$ and coincides with $g$. Since $v$ has no neighbour in $P_{1} \cap V_{j-1}$ it follows that for any edge $u w$ with $u \in P_{2}$ and $w \in P_{2}$, $|f(u)-f(w)| \leq d$. Also, $f^{\prime}$ satisfies point 2 of definition 2 because $\pi_{p+1}$ is the rightmost position in $P_{2} \cap \mathcal{B}_{j}$. Furthermore $f^{\prime}$ satisfies point 3 of Definition 2 by point 3 of Definition 3. Finally $f^{\prime}$ satisfies point 4 of Definition 2 by point 5 of Definition 3 .

Now we are ready to prove the main lemma of the section which allows us to obtain the desired result. 
Lemma 4 There is a pushing partial embedding $f: V_{J} \rightarrow \mathcal{B}_{J}$ with distortion at most $d$ such that $f$ agrees with $h$, coincides with $g$ and such that for every $j \in J, f\left(t_{j}\right)=l_{j}$ and no other vertex in $V_{j}$ is mapped before $t_{j}$ by $f$ if and only if there exists sequence of states $\zeta_{1}, \zeta_{1}, \ldots, \zeta_{\left|\mathcal{B}_{J}\right|}$ such that $(a) \zeta_{1}=(\emptyset, \emptyset, \emptyset, 0)$; (b) $\zeta_{i+1}$ succeeds $\zeta_{i}$ for all $i \in\left\{1, \ldots,\left|\mathcal{B}_{J}\right|-1\right\}$; and $(c) \zeta_{\left|\mathcal{B}_{J}\right|}=\left(V_{J}, X, Y,\left|\mathcal{B}_{J}\right|\right)$.

Proof: Let $f: V_{J} \rightarrow \mathcal{B}_{J}$ be a pushing partial embedding with distortion at most $d$ such that $f$ agrees with $h$, coincides with $g$ and such that for every $j \in J, f\left(t_{j}\right)=l_{j}$ and no other vertex in $V_{j}$ is mapped before $t_{j}$ by $f$. With the help of $f$ we define the sequence of feasible states as follows. For every $p \leq\left|\mathcal{B}_{J}\right|, P$ is the set of vertices $f$ maps to $\pi_{0}, \ldots, \pi_{p}, Q$ is the set of vertices in $P$ such that for every $j$ such that $P \cap V_{j} \neq \emptyset, Q$ contains exactly one vertex $q_{j}, f$ maps all vertices in $P \cap V_{j}$ to the left of $q_{j}$. Finally $R$ is the set of positions that $f$ maps the vertices of $Q$. The construction of the sequence of states implies that $\zeta_{1}=(\emptyset, \emptyset, \emptyset, 0)$, $\zeta_{i+1}$ succeeds $\zeta_{i}$ for all $i \in\left\{1, \ldots,\left|\mathcal{B}_{J}\right|-1\right\}$ and that $\zeta_{\left|\mathcal{B}_{J}\right|}=\left(V_{J}, X, Y,\left|\mathcal{B}_{J}\right|\right)$.

For the reverse direction suppose that we have sequence of feasible states $\zeta_{1}, \zeta_{1}, \ldots, \zeta_{\left|\mathcal{B}_{J}\right|}$ such that $\zeta_{1}=(\emptyset, \emptyset, \emptyset, 0)$; (b) $\zeta_{i+1}$ succeeds $\zeta_{i}$ for all $i \in\left\{1, \ldots,\left|\mathcal{B}_{J}\right|-1\right\}$; and (c) $\zeta_{\left|\mathcal{B}_{J}\right|}=$ $\left(V_{J}, X, Y,\left|\mathcal{B}_{J}\right|\right)$. Since $\zeta_{1}=(\emptyset, \emptyset, \emptyset, 0)$ is feasible Lemma 3 implies that $\zeta_{\left|\mathcal{B}_{J}\right|}=\left(V_{J}, X, Y,\left|\mathcal{B}_{J}\right|\right)$ is feasible as well. The definition of feasibility guarantees the existence of the desired $f$, concluding the proof.

Finally, we ready to proceed with the lemma used for the analysis of Step 3.

Lemma 5 There is an algorithm that for given $G, d, h, J, g$ and $T$ decides whether there is a pushing partial embedding $f: V_{J} \rightarrow \mathcal{B}_{J}$ with distortion at most d such that $f$ agrees with $h$, coincides with $g$ and such that for every $j \in J, f\left(t_{j}\right)=l_{j}$ and no other vertex in $V_{j}$ is mapped before $t_{j}$ by $f$ in time and space $2^{n} \cdot n^{O(|J|)}$.

Proof: As we observed already, the number of states is at most $2^{n} \times n^{|J|} \times(d+1)^{|J|} \times\left|\mathcal{B}_{j}\right| \leq$ $2^{n} \cdot n^{O(|J|)}$. The algorithm decides the existence of $f$ by applying Lemma 4 . The algorithm starts in the state $(\emptyset, \emptyset, \emptyset, 0)$ and does breadth first search on the graph where vertices are the states and there is a directed edge from a state $\zeta_{i}$ to a state $\zeta_{j}$ if $\zeta_{j}$ succeeds $\zeta_{i}$. We do not keep this graph explicitly and rather generate the vertices of this graph as and when required in our breadth first search. Whenever we are at state $\zeta$ we can find all possible successor states in polynomial time. By Lemma 4 there is a required embedding $f$ if and only if there is a path from $(\emptyset, \emptyset, \emptyset, 0)$ to $\left(V_{J}, X, Y,\left|\mathcal{B}_{J}\right|\right)$. Our algorithm needs $2^{n} \cdot n^{O(|J|)}$ space to keep track of the set of states visited by the breadth first search algorithm. Since the number of states is bounded by $2^{n} \cdot n^{O(|J|)}$ and the number of successors of a state is at most $d+2$ the number of vertices and edges in the state graph is upper bounded by $2^{n} \cdot n^{O(|J|)}$. Hence the algorithm takes $2^{n} \cdot n^{O(|J|)}$ time and space.

Observe that applying Lemma 5 together with the analysis presented for Algorithm EXACT-Dist over the previous section yields a running time bound of $6^{n+o(n)}$. In fact, our algorithm runs in time $5^{n+o(n)}$. The next section is devoted to proving this.

\subsection{A Refined Analysis}

In this section we prove that the total number of states ever produced by our algorithm is $5^{n+o(n)}$. Since the running time of the algorithm is proportional to the number of states we generate up to a subexponential factor, this implies that algorithm ExACT-DisT runs in time $5^{n+o(n)}$. 
Lemma 6 The algorithm described in the previous sections runs in time $5^{n+o(n)}$.

Proof: Let a super-state be a two-tuple $(h, \zeta)$ where $h$ is a bucket assignment and $\zeta$ is a state generated by algorithm EXACT-Dist. We say that a super-state is visited by the algorithm to decide whether there is an embedding $f$ of $G$ into the line with distortion at most $d$ if $\zeta$ is generated at a call to EXACT-DisT with $h$ as the required bucket assignment. The total running time of the algorithm is directly proportional to the total number of times each super state is visited. First we argue that each super state is visited at most $2^{o(n)}$ times. For a fixed $h, J=\{x, \ldots, y\}, T, l_{x} \ldots l_{y}$ and $g$ the state $(h, \zeta)$ is visited at most one. However the number of possible $J$ 's is $O\left(n^{2}\right),|T| \leq \frac{n}{\log ^{2} n}, y-x \leq|T|$ so the number of possible sets $T$ and $|J|$-tuples $l_{x}, \ldots, l_{y}$ is $2^{o(n)}$. Finally, in any recursive call the domain of $g$ is at most $\log ^{2} n \cdot \log \frac{n}{n / \log ^{2} n}=2 \log ^{2} n \log \log n$. Hence the number of possible $g$ 's is $2^{o(n)}$. Thus each super-state is visited at most $2^{o(n)}$ times.

For every fixed $h$ and $P \subseteq V(G)$ there are at most $2^{o(n)}$ triplets $(Q, R, p)$ such that the super-state $(h,(P, Q, R, p))$ is visited. This is true because $|Q| \leq n / \log ^{2} n,|R| \leq n / \log ^{2} n$ and $p$ is an integer in $\{1, \ldots, d n\}$.

Finally we need to argue that there are at most $n^{2} \cdot 5^{n}$ pairs $(h, P)$ such that there is a triplet $(Q, R, p)$ such that the super-state $(h,(P, Q, R, p))$ is visited. Let $T$ be the spanning tree of $G$ rooted at $r_{T}$ that we used to list bucket assignments of $G$. We prove that for a fixed integer interval $J \subseteq\{1, \ldots, n\}$ with $|J| \leq n / \log ^{2} n$ the number pairs $(h, P)$ such that there is a triplet $(Q, R, p)$ such that the super-state $(h,(P, Q, R, p))$ is visited by the algorithm during a call to algorithm EXACT-DisT with $J$ as parameter is at most $5^{n}$. Every such pair corresponds to a labelling of the tree $T$. The vertices of $T$ are labelled from the set $\{0,1\}$ with a vertex $v$ labelled 1 if $v \in P$. The edges of $T$ are labelled from the set $\{-1,0,1\}$ such that for every $u v \in E(T)$ where $u$ is a parent of $v$, the edge $u v$ is labelled $h(v)-h(u)$. For a subtree $T^{\prime}$ of $T$ rooted at $r_{T}$ we say that a labelling $L$ of $T^{\prime}$ is good if there is a super-state visited by the algorithm whose labelling restricted to $T^{\prime}$ is exactly $L$. We prove that if $T^{\prime}$ has $n^{\prime}$ vertices then the number of good labellings of $T^{\prime}$ is at most $5^{n^{\prime}}$ by induction on $n^{\prime}$. If $n^{\prime}=1$ this follows trivially. Suppose now that the assertion holds for some $n^{\prime}$ and consider a subtree $T^{\prime}$ on $n^{\prime}+1$ vertices. Let $l$ be a leaf of $T^{\prime}$. Notice that any good labelling of $T^{\prime}$ restricted to $T^{\prime} \backslash l$ is a good labelling of $T^{\prime} \backslash l$. By the induction hypothesis there are at most $5^{n^{\prime}}$ good labellings of $T^{\prime} \backslash l$. We prove that there are at most 5 ways to extend a good labelling $L$ of $T^{\prime} \backslash l$ to a good labelling of $T^{\prime}$.

Let $l^{\prime}$ be the parent of $l$ in $T$ and let $P_{l^{\prime}}$ be the path from $r_{T}$ to $l^{\prime}$ in $T^{\prime}$. Let $z=$ $\sum_{u v \in E\left(P_{l^{\prime}}\right)} L(u v)$. If $z-1 \notin J, z \notin J$ or $z+1 \notin J$ we prove that there are only 5 ways to extend $L$ to a good labelling of $T^{\prime}$. In order to extend $L$ we need to specify $L\left(l l^{\prime}\right)$ and $L(l)$. Notice that if $z+L\left(l l^{\prime}\right) \notin J$ then $L(l)$ must be 0 in any good labelling. Thus in this case there are at most 5 ways to extend $L$. Now, consider the case that $\{z-1, z, z+1\} \subseteq J$ and $L\left(l^{\prime}\right)=0$. Then if $L\left(l l^{\prime}\right)=-1$ then $L(l)$ can not be 1 in a good labelling. Finally, consider the case that $\{z-1, z, z+1\} \subseteq J$ and $L\left(l^{\prime}\right)=1$. Then if $L\left(l l^{\prime}\right)=1$ then $L(l)$ can not be 0 in a good labelling. In both these cases there are at most 5 ways to extend $L$, concluding the proof.

We conclude with the following theorem.

Theorem 1 There is an algorithm that given a graph $G$ on $n$ vertices constructs a noncontracting embedding of the shortest path metric generated by $G$ into the line with minimum distortion in time $5^{n+o(n)}$ and space $2^{n+o(n)}$. 


\section{Concluding remarks and open problems}

In this paper we have provided the first single vertex exponential time algorithm for computing a minimum distortion embedding of a graph metric into the line. This result gives rise to many challenging questions.

How fast is it possible to compute a minimum distortion embedding of a graph $G$ into the metric of another graph $H$ ? Is there a $2^{O(|V(G)|)}$ time algorithm for this problem, or can one show that this is impossible up to some complexity theoretic assumption? How does the problem behave if the host graph $H$ is a tree? Even when $H$ is a binary tree, this does not seem to be an easy problem. At a first glance it would seem that our algorithm should be directly extendable to find a minimum distortion embedding of a graph $G$ into a given cycle $C$. However this does not look to be easy and we leave it as an open problem whether finding a minimum distortion embedding of a graph $G$ into a given cycle $C$ can be done in $2^{O(|V(G)|)}$ time.

We believe that the world of embeddings provides a lot of challenges to the area of moderately exponential time algorithms and is worth to be explored. We hope that our result will lead to further investigation of the combinatorially challenging field of embeddings within the framework of moderately exponential time algorithms.

\section{References}

[1] M. Bădoiu, J. Chuzhoy, P. Indyk, And A. Sidiropoulos, Low-distortion embeddings of general metrics into the line, in Proceedings of the 37th Annual ACM Symposium on Theory of Computing (STOC), ACM, 2005, pp. 225-233.

[2] M. Bădoiu, K. Dhamdhere, A. Gupta, Y. Rabinovich, H. Räcke, R. Ravi, and A. Sidiropoulos, Approximation algorithms for low-distortion embeddings into low-dimensional spaces, in Proceedings of the 16th Annual ACM-SIAM Symposium on Discrete Algorithms (SODA), SIAM, 2005, pp. 119-128.

[3] M. Badoiu, P. Indyk, And A. Sidiropoulos, Approximation algorithms for embedding general metrics into trees, in Proceedings of the 18th Annual ACM-SIAM Symposium on Discrete Algorithms (SODA), ACM and SIAM, 2007, pp. 512-521.

[4] M. Cygan and M. Pilipczuk, Faster exact bandwidth, in Proceedings of the 34th International Workshop on Graph-Theoretic Concepts in Computer Science (WG 2008), vol. 5344 of Lecture Notes in Computer Science, 2008, pp. 101-109.

[5] U. FeIGe, Coping with the NP-hardness of the graph bandwidth problem, in Proceedings of the 7th Scandinavian Workshop on Algorithm Theory (SWAT), vol. 1851 of LNCS, Springer, Berlin, 2000, pp. 1019.

[6] M. R. Fellows, F. V. Fomin, D. Lokshtanov, E. Losievskaja, F. A. Rosamond, and S. Saurabh, Parameterized low-distortion embeddings - graph metrics into lines and trees, CoRR, abs/0804.3028 (2008).

[7] A. Gupta, I. Newman, Y. Rabinovich, And A. Sinclair, Cuts, trees and $l_{1}$-embeddings of graphs, Combinatorica, 24 (2004), pp. 233-269.

[8] P. INDYK, Algorithmic applications of low-distortion geometric embeddings, in Proceedings of the 42nd IEEE Symposium on Foundations of Computer Science (FOCS), IEEE, 2001, pp. 10-33.

[9] C. Kenyon, Y. Rabani, and A. Sinclair, Low distortion maps between point sets, in Proceedings of the 36th Annual ACM Symposium on Theory of Computing (STOC), ACM, 2004, pp. 272-280.

[10] N. Linial, Finite metric-spaces-combinatorics, geometry and algorithms, in Proceedings of the International Congress of Mathematicians, Vol. III, Beijing, 2002, Higher Ed. Press, pp. 573-586. 